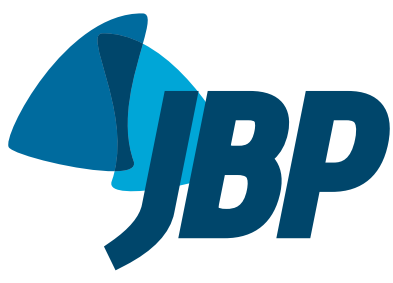

\title{
Open letter to city, state, and federal health authorities, to State Health Councils, and to the National Council of Municipal Health Secretaries in Brazil
}

The World Health Organization estimates that chronic respiratory diseases (CRDs) are responsible for approximately 12 million deaths annually. Of the five leading causes of death worldwide, three are lung diseases. In Brazil, estimates indicate that approximately $15 \%$ of the population has a CRD, and CRDs are therefore the cause of approximately 200,000 hospitalizations. In the state of Minas Gerais, CRDs as a group are the third leading contributor to the disability burden. One example is COPD, which was projected to rank third among the leading causes of death only in 2030 and was already ranked as such in 2014. In Brazil, COPD affects nearly $16 \%$ of the population over 40 years of age.

The Brazilian Sistema Único de Saúde (SUS, Unified Health Care System) has introduced a series of public policies and initiatives aimed at addressing CRDs as a group, including the following: primary care booklets; clinical protocols and therapeutic guidelines for asthma; programs for the management and control of tuberculosis and smoking; the Popular Pharmacy program, which distributes essential medications and high-cost drugs at no charge, with support from state and federal funding; and the prevention, diagnosis, and treatment of bronchopulmonary neoplasms.

CRDs are not explicitly part of the program that has recently been launched by the Brazilian National Ministry of Health, called "Programa Mais Especialidades" (More Specialties Program). This shows the lack of coordination among such initiatives, which is why they have not produced the necessary response to the multifaceted epidemiological context involving them. Therefore, it is imperative that strategies for organically promoting their integration be proposed.

The strategy that has been implemented in the city of Belo Horizonte and its metropolitan area, which was presented and discussed in a workshop held on November 27, 2015 and attended by professionals from the four corners of Brazil who have extensive experience in the management of CRDs at the level of SUS, was based on the recommendations of the World Health Organization Practical Approach to Lung Health program and led to the publication, on July 1, 2014, of Resolution No. 1,861 of the Comissão Intergestores Bipartite de Minas Gerais (Bipartite Liaison Committee of Minas Gerais)-SUS/ MG, approving the establishment of a management program specifically aimed at CRDs as a group. That resolution, which would allow the implementation of a comprehensive health care model that would bring together primary health care facilities, specialty centers, hospitals (including those affiliated with universities), and other existing resources, highlighting the complexity, severity, and level of resolution of CRDs at the various levels of health care, has yet to be put into practice. We emphasize the previous successful experience in care provision to patients with CRDs in the city of Ribeirão das Neves, located in the state of Minas Gerais, which was based on the recommendations of the aforementioned Practical Approach to Lung Health program and led to effective care, as well as increasing the feasibility of the activities. It is of note that, in the other Brazilian states (including the Federal District of Brasília), there is no formalized proposal with this level of comprehensiveness, a fact that underscores the urgent need to develop a strategy to be implemented nationwide.

The professionals working in civil society organizations, medical societies, the legislature, universities, and city/ state administration who met in Belo Horizonte draw attention to the lack of articulation among the current public policies aimed at CRDs and demand that public health managers urgently adopt integrating strategies that are based on existing experiences-the focus of the event held on that date-and, at the same time, make themselves available to city, state, and federal health authorities to develop and implement a comprehensive line of care for treatment of CRDs. That line of treatment should be given the same priority as that now given to other diseases of equal epidemiological importance that affect the Brazilian population.

Belo Horizonte, February 1, 2016.

Board of Representatives for the Global Initiative for Chronic Obstructive Lung Disease in Brazil

Board of Representatives for the Global Initiative for Asthma in Brazil

Board of Representatives for the World Health Organization Global Alliance against Chronic Respiratory Diseases in Brazil

Medical Association of Minas Gerais

Minas Gerais Society of Pulmonology and Thoracic Surgery Federal University of Minas Gerais Hospital das Clínicas Health Committee of the Legislative Assembly of Minas Gerais 